\title{
Giant Hysteresis of Single-Molecule Magnets Adsorbed on a Nonmagnetic Insulator
}

\author{
Christian Wäckerlin, Fabio Donati, Aparajita Singha, Romana Baltic, Stefano Rusponi, \\ Katharina Diller, François Patthey, Marina Pivetta, Yanhua Lan, Svetlana Klyatskaya, \\ Mario Ruben, Harald Brune, and Jan Dreiser*
}

Single-molecule magnets $(\mathrm{SMMs})^{[1]}$ are very promising for molecular spintronics ${ }^{[2]}$ and quantum information processing, ${ }^{[3]}$ because of their magnetic bistability and the quantum nature of their spin. The first step toward devices based on SMMs is their adsorption onto electrode surfaces..$^{[4,5]}$ However, this step already represents a serious obstacle as it severely compromises the magnetic remanence. ${ }^{[6-13]}$ Here, we solve this problem by introducing a tunnel barrier between the SMMs and the metal electrode. For $\mathrm{TbPc}_{2} \mathrm{SMMs}^{[14,15]}$ on nonmagnetic, insulating $\mathrm{MgO}$ on $\mathrm{Ag}(100)$ we demonstrate record values of the magnetic remanence and the hysteresis opening, outperforming any previously reported surface adsorbed SMMs.

The two key properties of a magnet relevant to devices are large remanence and wide hysteresis opening. Achieving these goals represents a largely unresolved challenge for SMMs adsorbed at surfaces. Current strategies are to exploit weak adsorption, e.g., on graphite, ${ }^{[8,9]}$ or decoupling from the surface by long chemical linkers ${ }^{[4,5,10]}$ or bulky ligands..$^{[11,12]}$ While some of the approaches were successful in achieving a sizeable butterfly-like hysteresis opening, ${ }^{[4,5,10-12]}$ so far all attempts to enhance the vanishingly small magnetic remanence of SMMs in contact with surfaces have failed. ${ }^{[4-13]}$ Consequently, the magnetic remanence of surface-adsorbed SMMs lags far behind the benchmark set by bulk samples, ${ }^{[16]}$ which are, however, not useful for device applications.

Here we introduce an entirely different strategy, namely, the insertion of a tunnel barrier between the SMMs and the

Dr. C. Wäckerlin, Dr. F. Donati, A. Singha, R. Baltic, Dr. S. Rusponi, Dr. K. Diller, Dr. F. Patthey, Dr. M. Pivetta, Prof. H. Brune, Dr. J. Dreiser Institute of Physics (IPHYS)

École Polytechnique Fédérale de Lausanne (EPFL)

Station 3, CH-1015 Lausanne, Switzerland

E-mail: jan.dreiser@psi.ch

Dr. Y. Lan, Dr. S. Klyatskaya, Prof. M. Ruben

Institute of Nanotechnology

Karlsruhe Institute of Technology (KIT)

D-76344 Eggenstein-Leopoldshafen, Germany

Prof. M. Ruben

Institut de Physique et Chimie des Matériaux (IPCMS)

Université de Strasbourg

F-67034 Strasbourg, France

Dr. J. Dreiser

Swiss Light Source

Paul Scherrer Institut (PSI)

$\mathrm{CH}-5232$ Villigen, Switzerland

DOI: 10.1002/adma.201506305 metal electrode. We use nonmagnetic, insulating $\mathrm{MgO}$, wellknown in inorganic spintronic applications, ${ }^{[17,18]}$ which allows to control the electron tunneling rate over many orders of magnitude. ${ }^{[19]}$ Moreover, we employ the $\mathrm{TbPc}_{2} \mathrm{SMM}^{[14,15,20-23]}$ as a model system. In the neutral molecule, the $\mathrm{Tb}(\mathrm{III})$ ion exhibits an electronic spin state of $J=6$. It is sandwiched between two phthalocyanine (Pc) macrocycles (cf. schematic view in Figure 1a) hosting an unpaired electron delocalized over the Pc ligands. The easy-axis-type magnetic anisotropy imposes an energy barrier of $\approx 65 \mathrm{meV}$ for magnetization reversal, ${ }^{[23]}$ which is largest within the whole series of lanthanide-Pc SMMs. $^{14,15]}$ On nonmagnetic conducting substrates, only vanishing remanence ${ }^{[6-10]}$ and very narrow hysteresis loops ${ }^{[6-9]}$ were observed, much smaller than in bulk measurements, ${ }^{[20]}$ illustrating the disruptive effects of the surface. We note that the adsorption of $\mathrm{TbPc}_{2}$ on (anti)ferromagnetic materials represents a different situation because of the magnetic exchange interaction with the substrate. ${ }^{[24,25]}$ In those cases, the SMMs were not shown to exhibit slow relaxation of magnetization. Rather, the hysteresis is linked to the one of the magnetic substrates, i.e., it is not an intrinsic property of the SMMs. Overall, the detailed knowledge on $\mathrm{TbPc}_{2}$ makes it an ideal candidate to test if a tunnel barrier can boost the magnetic properties of surface-adsorbed SMMs. In this communication we show that the magnetic remanence and hysteresis opening obtained with $\mathrm{TbPc}_{2}$ on $\mathrm{MgO}$ tunnel barriers outperform the ones of any other surface-adsorbed $\mathrm{SMM}^{[4-13,26]}$ as well as those of bulk samples of $\mathrm{TbPc}_{2} \cdot{ }^{[20]}$

The scanning tunneling microscopy (STM) images in Figure $1 \mathrm{~b}, \mathrm{c}$ show that $\mathrm{TbPc}_{2}$ self-assembles by forming perfectly ordered 2D islands on two monolayers (MLs) of $\mathrm{MgO}$ on $\mathrm{Ag}(100)$. In line with former results, the SMMs are adsorbed flat on the surface (cf. discussion of our STM and X-ray linear dichroism (XLD) data below). ${ }^{[6,27]}$ This excludes that the extraordinary magnetic properties observed in this study are due to upstanding molecules having their macrocycles perpendicular to the surface, which would lead to a reduced interaction of the $\mathrm{Tb}(\mathrm{III})$ ion with the surface. The high-resolution image in Figure 1c reveals eight lobes per molecule, reminiscent of the staggered conformation of the two phthalocyanine ligands. ${ }^{\text {[27] }}$ Islands with the identical molecular assembly are formed by $\mathrm{TbPc}_{2}$ adsorbed directly onto $\mathrm{Ag}(100)$, as shown in the Supporting Information.

The magnetic properties of the $\mathrm{Tb}(\mathrm{III})$ ions in the surfaceadsorbed SMMs are determined by X-ray magnetic circular dichroism (XMCD) measurements at the $\mathrm{M}_{4,5}(3 d \rightarrow 4 f)$ edges of $\mathrm{Tb}$. For sub-MLs of $\mathrm{TbPc}_{2}$ on $\mathrm{MgO}$ we find a strong remanence larger than $40 \%$ of the saturation magnetization $M_{\text {sat }}$ and 
a

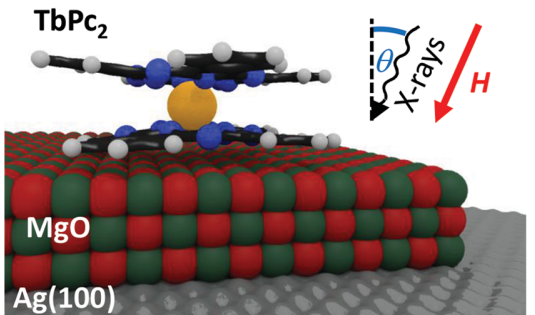

b

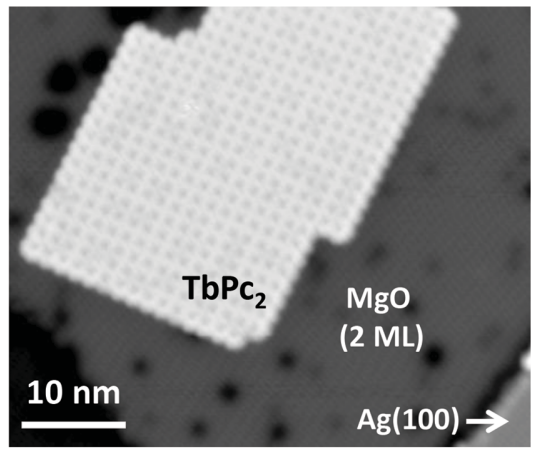

C

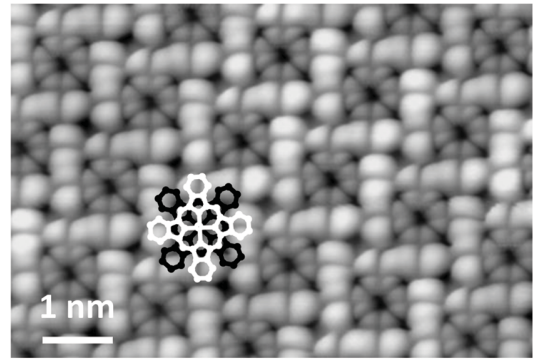

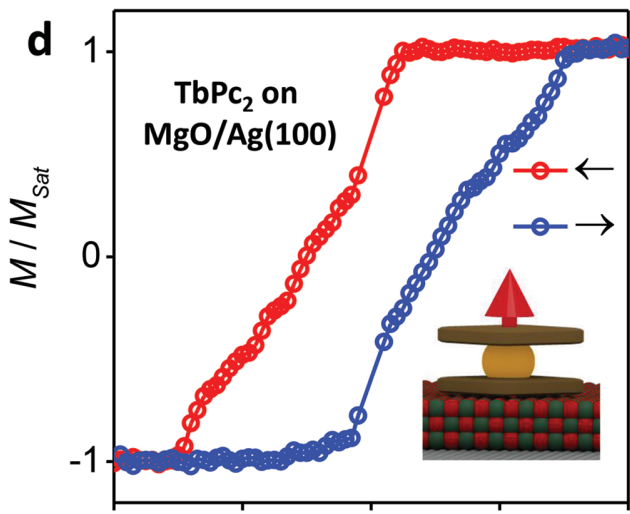

e
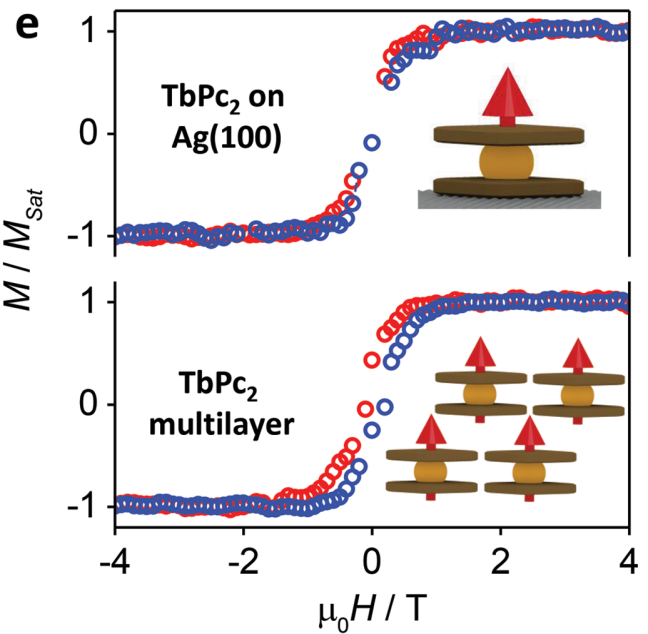

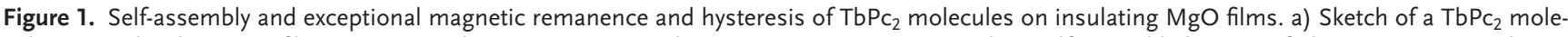
cule on an ultrathin $\mathrm{MgO}$ film on $\mathrm{Ag}(100)$. b,c) Scanning tunneling microscopy images revealing self-assembled arrays of $\mathrm{TbPc}_{2}$ on two monolayers (MLs) of MgO. The image sizes and parameters (scale bar, bias voltage, and current setpoint) are $(10 \mathrm{~nm},+2 \mathrm{~V}, 20 \mathrm{pA})$ for (b) and (1 nm, $-2 \mathrm{~V}, 20 \mathrm{pA})$ for (c), respectively. $\mathrm{d}$,e) Hysteresis loops obtained with XMCD at $3 \mathrm{~K}$ for $0.6 \mathrm{ML} \mathrm{TbPc_{2 }}$ on $5 \mathrm{ML} \mathrm{MgO}$ compared with $0.3 \mathrm{MLTbPc}_{2}$ adsorbed directly on $\mathrm{Ag}(100)$ and with a $\mathrm{TbPc}_{2}$ multilayer ( $3 \mathrm{ML}$ ) on $\mathrm{MgO}$ (field sweep rate $2 \mathrm{~T} \mathrm{~min}^{-1}$, normal incidence, X-ray flux (d) $0.25 \Phi_{0}$ and (e) $\Phi_{0}$, respectively).

a hysteresis opening up to $3 \mathrm{~T}$ at $3 \mathrm{~K}$ (Figure 1d). These values vastly exceed the corresponding records reported for any surface-adsorbed $\mathrm{SMM}^{[4-13,26]}$ as well as the ones reported for bulk $\mathrm{TbPc}_{2} \cdot{ }^{[20]}$ The large remanence indicates that quantum tunneling of magnetization is strongly suppressed for fields below $3 \mathrm{~T}$, with only a very subtle modulation of the relaxation rate across the hysteresis loop.

The effect of the tunnel barrier becomes evident when comparing with $\mathrm{TbPc}_{2}$ directly adsorbed onto $\mathrm{Ag}(100)$ where the hysteresis opening is barely visible. In fact, the area of the opening has decreased by a factor of 10 (Figure 1e). The large opening of sub-MLs on $\mathrm{MgO} / \mathrm{Ag}(100)$ is also reduced in $\mathrm{TbPc}_{2}$ multilayers (Figure 1e). This is attributed to magnetic interactions between the molecules. ${ }^{[21]}$ In addition to $\mathrm{MgO}$, we also investigated hexagonal boron nitride $(h-\mathrm{BN})^{[28]}$ as a tunnel barrier. The hysteresis opening on $h$-BN is wider than the one reported for most surface-adsorbed $\mathrm{TbPc}_{2}$ (Supporting Information), however, it is significantly narrower than on the $\mathrm{MgO}$ thin film. $\mathrm{MgO}$ is more efficient in suppressing electron scattering from the substrate as it can be grown in multilayers, while $h$-BN forms a self-limiting monolayer.
The electronic ground state and magnetic moments of the $\mathrm{Tb}$ ion as well as the molecular orientation are inferred from the X-ray absorption spectra (XAS) and their circular (XMCD) and linear (XLD) dichroism (Figure 2). XLD, which is directly sensitive to the molecular orientation, evidences that the molecules adsorb with the same orientation on $\mathrm{MgO}$ (Figure 2) and on $\mathrm{Ag}(100)$ (Supporting Information). This is in line with our STM results (Figure 1b,c and Figures S1-S3, Supporting Information) showing that the $\mathrm{TbPc}_{2}$ macrocycles are parallel to the surfaces of $\mathrm{MgO}$ and $\mathrm{Ag}(100)$. Furthermore, our results are consistent with XLD spectra of $\mathrm{TbPc}_{2}$ on metal surfaces reported in the literature. ${ }^{[6,7]}$ The same orientation is also observed for $\mathrm{TbPc}_{2}$ on $h$-BN and for the multilayer (Supporting Information). The $\mathrm{Tb}$ spin and orbital magnetic moments extracted from the XMCD spectra (cf. Supporting Information) are in excellent agreement with previous studies of $\mathrm{TbPc}_{2}$ on metal surfaces ${ }^{[6,7]}$ and on graphite. ${ }^{[8,9]}$ Therefore the larger remanence is neither due to a different magnetic ground state of the $\mathrm{Tb}$ ion nor to strong modifications in the magnetic anisotropy.

To determine the magnetic relaxation times of $\mathrm{TbPc}_{2} / \mathrm{MgO}$, we have performed time-dependent XMCD measurements at $0.5 \mathrm{~T}$ after saturating the magnetization at $4 \mathrm{~T}$ (Figure 3). 

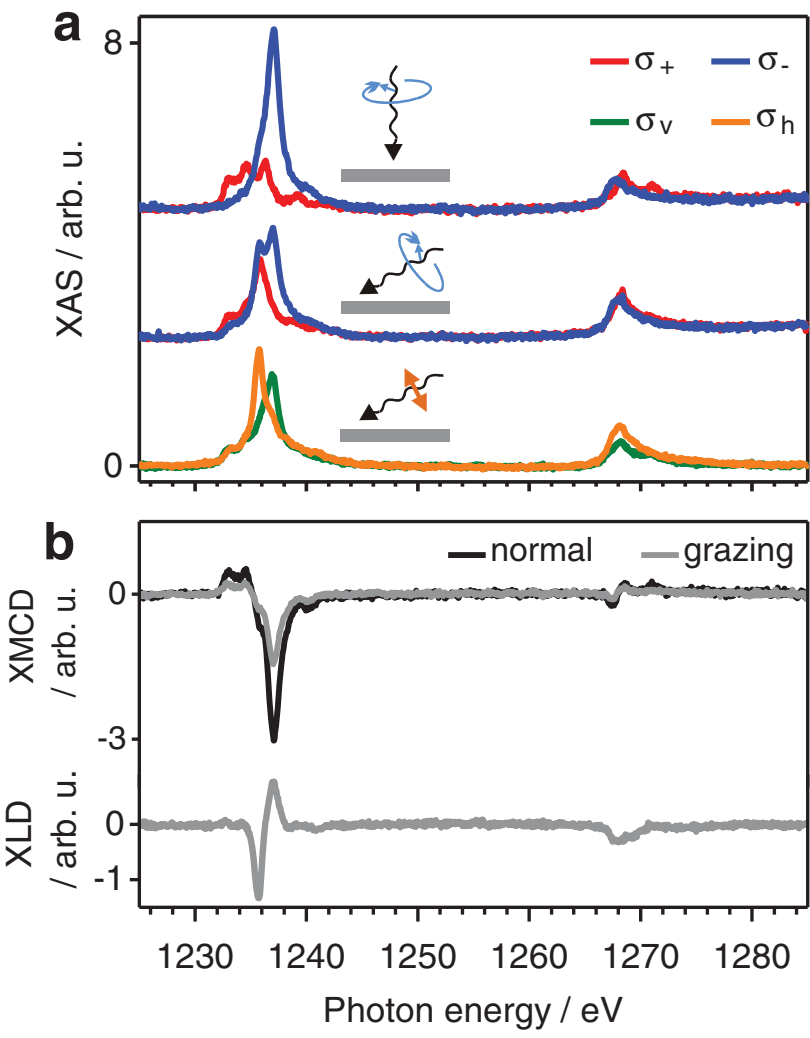

Figure 2. X-ray spectra of a submonolayer of $\mathrm{TbPc}_{2}$ on $\mathrm{MgO}$ revealing the magnetic anisotropy and the orientation of the molecules. X-ray absorption spectra (XAS) at the $\mathrm{Tb}_{4,5}$ edges acquired at $3 \mathrm{~K}$ using circularly $\left(\sigma^{+}, \sigma^{-}\right)$and linearly $\left(\sigma^{h}, \sigma^{v}\right)$ polarized light. The same arbitrary units are used in (a) and (b). a) The spectra with circularly polarized X-rays were obtained in normal $\left(\theta=0^{\circ}\right)$ and grazing $\left(\theta=60^{\circ}\right)$ incidence in an applied magnetic field of $6.8 \mathrm{~T}$. b) Their difference, XMCD, is a direct measure of the magnetic moment of Tb. The peak XMCD-to-XAS ratio $\left(\sigma^{+}-\sigma^{-}\right) /\left(\sigma^{+}+\sigma^{-}\right)$is $-80 \%$ and $-55 \%$ for normal and grazing incidence, respectively. The X-ray linear dichroism (XLD), the difference of the linearly polarized XAS, is obtained at $50 \mathrm{mT}$ in grazing incidence, with the strongest XLD-to-XAS ratio of $-45 \%$.

The magnetization versus time traces $M(t)$ decay exponentially (Figure 3a). This decay with rate $\tau^{-1}$ becomes faster with increasing X-ray flux. Therefore, intrinsic relaxation
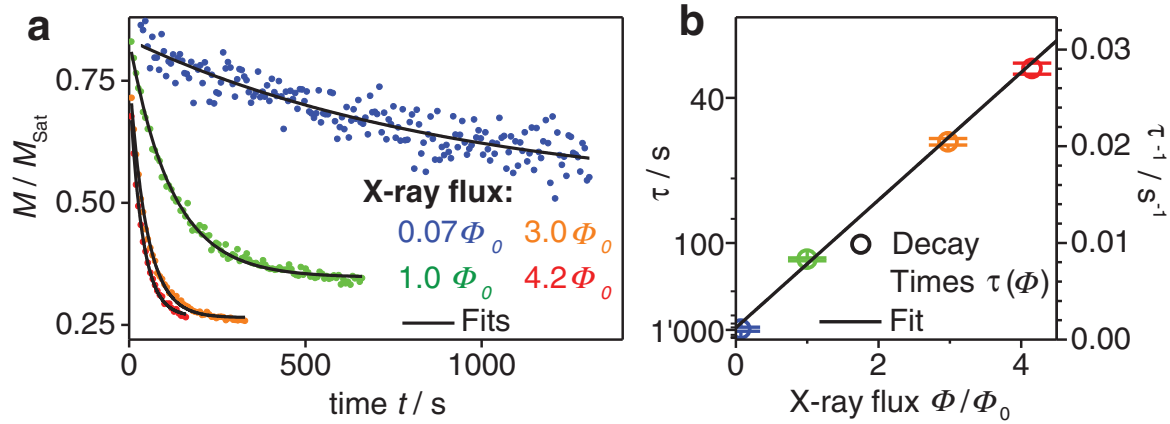

Figure 3. Intrinsic relaxation and X-ray induced demagnetization. The time-dependent XMCD signal was obtained at $0.5 \mathrm{~T}$ after magnetizing the samples at $4 \mathrm{~T}$ and switching on the X-ray beam at time $t=0$, immediately after reaching $0.5 \mathrm{~T}$. a) With increasing $\mathrm{X}$-ray flux $\Phi$, the magnetization $M$ decays faster. The exponential fits yield the decay times $\tau$ of the magnetization as a function of X-ray flux $\Phi$. b) The intercept yields the intrinsic relaxation time $\tau_{\mathrm{i}}=14_{-4}^{+10} \mathrm{~min}$. (rate $\tau_{\mathrm{i}}^{-1}$ ) and photon-induced demagnetization (rate $\left.\tau_{\mathrm{ph}}^{-1}\right)^{[29]}$ coexist. The respective rates add up yielding the decay rate $\tau^{-1}=\tau_{\mathrm{i}}^{-1}+\tau_{\mathrm{ph}}^{-1}$. Consistently, the fit $\tau(\Phi)^{-1}=\tau_{\mathrm{i}}^{-1}+\sigma \Phi \quad$ in Figure $3 \mathrm{~b}$ shows that the decay rates are linear with the X-ray flux $\Phi$ within the error bars. The intercept at zero X-ray flux yields the intrinsic relaxation time $\tau_{\mathrm{i}}=14_{-4}^{+10} \mathrm{~min}$ at $0.5 \mathrm{~T}$, and the slope $\sigma=0.21 \pm 0.05 \mathrm{~nm}^{2}=(2.1 \pm 0.5) \times 10^{9}$ barn is the cross section of the photon-induced demagnetization process. The asymptotic values of the magnetization decrease with increasing X-ray flux $\Phi$, indicating that the X-ray-induced demagnetization drives the magnetization to a value which is lower than the thermodynamic equilibrium at $0.5 \mathrm{~T}$, in contrast to the intrinsic relaxation. Notably, the demagnetization is not a result of simple spatially homogeneous heating or radiation damage (cf. Supporting Information).

Temperature-dependent hysteresis loops of $\mathrm{TbPc}_{2} / \mathrm{MgO}$ (Figure 4) evidence slow relaxation of the magnetization beyond $6 \mathrm{~K}$. In fact, the hysteresis area still exhibits a finite value at $8 \mathrm{~K}$, which is the highest blocking temperature ever reported for surface-adsorbed SMMs.

We rationalize the large magnetic remanence and the wide hysteresis opening of $\mathrm{TbPc}_{2}$ on $\mathrm{MgO}$ by identifying two key aspects. These are, first, the strong suppression of scattering of conduction electrons from the metal at the molecule and, second, the low molecule-surface hybridization. The electron tunneling rate depends exponentially on the barrier thickness. For $\mathrm{MgO}$ tunnel barriers it is reduced by a factor of $\approx 3 \times 10^{3}$ per nanometer $(\approx 5 \mathrm{ML} \mathrm{MgO}) .{ }^{[30]}$ In accordance, narrower hysteresis loops are observed for thinner $\mathrm{MgO}$ and for $h$ - $\mathrm{BN}$ monolayers (cf. Supporting Information). This suggests that for thicker MgO films or bulk $\mathrm{MgO}$ the remanence and the hysteresis opening will be equal to or larger than the ones observed in the present study.

Regarding the second key aspect, bulk studies have shown that the preservation of the ideal $D_{4 \mathrm{~d}}$ symmetry is important to achieve long relaxation times and large coercive fields in $\mathrm{TbPc}_{2} \cdot{ }^{[21,22]}$ Symmetry breaking enables mixing terms in the $\mathrm{TbPc}_{2}$ spin Hamiltonian that, together with the hyperfine interaction, promotes quantum tunneling of magnetization especially around zero field. ${ }^{[4,5,21,22]}$ Owing to the low hybridization on $\mathrm{MgO}$ the upper and lower Pc ligands retain the same electronic structure as in the gas phase, preserving the molecular $D_{4 \mathrm{~d}}$ symmetry nearly perfectly. By contrast, upon direct adsorption onto metal surfaces the electronic structure of the lower Pc ligand in contact with the surface is slightly altered because of adsorption bonds and molecule-surface charge transfer, reducing the symmetry of the $\mathrm{Tb}$ ligand field to $C_{4 \mathrm{v}}$ or lower. Thus, symmetry breaking of the ligand field, presumably together with electron scattering, leads to the barely open hysteresis loops of $\mathrm{TbPc}_{2}$ on $\mathrm{Ag}(100)$ in this work and on $\mathrm{Au}(111),{ }^{, 7]}$ and to the closed loop on $\mathrm{Cu}(100){ }^{[6]}$ Graphite is weakly hybridizing, however, it does not suppress electron scattering leading to narrow hysteresis openings as well. ${ }^{[8,9]}$ 


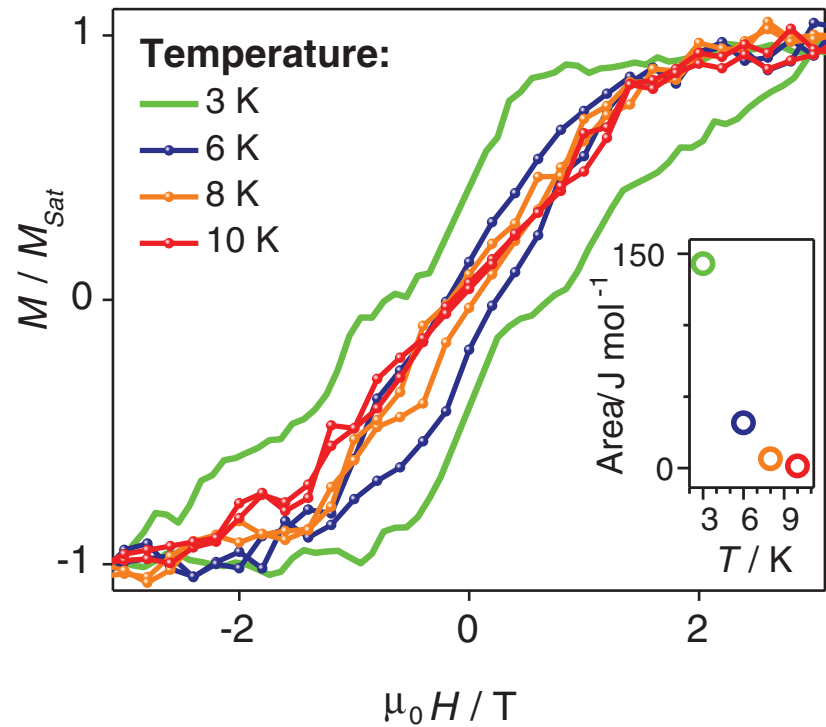

Figure 4. Temperature-dependent magnetization curves of $\mathrm{TbPc}_{2} / \mathrm{MgO} /$ $\mathrm{Ag}(100)$. With increasing temperature the magnetization loop gradually closes until the hysteresis fully vanishes at $10 \mathrm{~K}$ (X-ray flux $\Phi_{0}, 0.3 \mathrm{ML}$ $\mathrm{TbPc}_{2}$ on 4.8 ML MgO). The area of the hysteresis opening is plotted in the inset.

Concerning magnetic interactions between adjacent molecules for submonolayers of $\mathrm{TbPc}_{2}$, we observe a negligible influence of the molecular coverage on the hysteresis opening (Supporting Information). Together with the 2D molecular selfassembly seen by STM, this implies that lateral magnetic interactions are insignificant. On the contrary, our data on $\mathrm{TbPc}_{2}$ multilayers on $\mathrm{MgO}$ reveal that vertical interactions accelerate magnetization relaxation. ${ }^{[20,21]}$

In summary, we have demonstrated that $\mathrm{MgO}$ thin films realize the combination of efficient protection from electron scattering and weak molecule-surface hybridization to achieve optimal properties of SMMs on electrode surfaces. In addition, in the present case of $\mathrm{TbPc}_{2}$ the molecules are self-assembled into well-ordered islands leading to highly uniform molecular ensembles with out-of-plane easy axes. Epitaxial MgO layers promote a very large tunnel magnetoresistance in inorganic devices based on ferromagnetic electrodes. ${ }^{[31]}$ Therefore, the combination of SMMs and epitaxial $\mathrm{MgO}$ tunnel junctions opens up a path toward SMM-based tunnel devices.

\section{Experimental Section}

Sample Preparation: The $\mathrm{Ag}(100)$ single crystal substrate was prepared by repeated cycles of sputtering with $\mathrm{Ar}^{+}$ions and annealing. The epitaxial $\mathrm{MgO}$ layers were grown by sublimation of $\mathrm{Mg}$ in $\mathrm{O}_{2}$

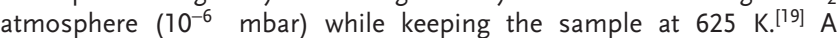
submonolayer of $\mathrm{TbPc}_{2}$ was sublimed at $650 \mathrm{~K}$ at a rate of $\approx 0.1 \mathrm{ML} \mathrm{min}{ }^{-1}$ onto the surface kept at room temperature. The multilayer sample was prepared by sublimation of $\approx 3 \mathrm{ML}$ of $\mathrm{TbPc}_{2}$ onto $\mathrm{MgO} / \mathrm{Ag}(100)$.

$X$-Ray Absorption Spectroscopy: The X-ray absorption experiments were performed at the EPFL/PSI X-Treme beamline ${ }^{[32]}$ at the Swiss Light Source at a temperature of $3 \mathrm{~K}$ in total electron yield mode using circularly $\left(\sigma^{+}, \sigma^{-}\right)$and linearly polarized $\left(\sigma^{h}, \sigma^{\nu}\right)$ X-rays with the magnetic field applied parallel to the X-ray beam. XMCD and XLD spectra correspond to the differences, $\left(\sigma^{+}-\sigma^{-}\right)$and $\left(\sigma^{\vee}-\sigma^{h}\right)$, respectively. The
X-ray flux was measured with a photodiode located after the last optical element of the beamline and was given in units of $\Phi_{0}=0.0034$ photons $\mathrm{nm}^{-2} \mathrm{~s}^{-1}$ (cf. extended methods in the Supporting Information).

\section{Supporting Information}

Supporting Information is available from the Wiley Online Library or from the author.

\section{Acknowledgements}

C.W., A.S., R.B., and J.D. gratefully acknowledge funding by the Swiss National Science Foundation (Grants PZ00P2_142474, 200020_157081/1 and 200021_146715/1). K.D. acknowledges support from the "EPFL Fellows" program co-funded by Marie Curie, FP7 grant agreement no. 291771. Y.L. and M.R. would like to thank the EC-FET-Open project "MOQUAS" and the ANR "MolQuSpin". The authors thank Christopher Bergman for the help with preparing the table of contents graphic.

Received: December 18, 2015

Revised: April 2, 2016

Published online: May 9, 2016

[1] R. Sessoli, D. Gatteschi, A. Caneschi, M. A. Novak, Nature 1993, $365,141$.

[2] M. Urdampilleta, S. Klyatskaya, J.-P. Cleuziou, M. Ruben, W. Wernsdorfer, Nat. Mater. 2011, 10, 502.

[3] S. Thiele, F. Balestro, R. Ballou, S. Klyatskaya, M. Ruben, W. Wernsdorfer, Science 2014, 344, 1135.

[4] M. Mannini, F. Pineider, P. Sainctavit, C. Danieli, E. Otero, C. Sciancalepore, A. M. Talarico, M.-A. Arrio, A. Cornia, D. Gatteschi, R. Sessoli, Nat. Mater. 2009, 8, 194.

[5] M. Mannini, F. Pineider, C. Danieli, F. Totti, L. Sorace, P. Sainctavit, M.-A. Arrio, E. Otero, L. Joly, J. C. Cezar, A. Cornia, R. Sessoli, Nature 2010, 468, 417.

[6] S. Stepanow, J. Honolka, P. Gambardella, L. Vitali, N. Abdurakhmanova, T.-C. Tseng, S. Rauschenbach, S. L. Tait, V. Sessi, S. Klyatskaya, M. Ruben, K. Kern, J. Am. Chem. Soc. 2010, 132, 11900.

[7] L. Margheriti, D. Chiappe, M. Mannini, P.-E. Car, P. Sainctavit, M.-A. Arrio, F. B. de Mongeot, J. C. Cezar, F. M. Piras, A. Magnani, E. Otero, A. Caneschi, R. Sessoli, Adv. Mater. 2010, 22, 5488.

[8] M. Gonidec, R. Biagi, V. Corradini, F. Moro, V. De Renzi, U. del Pennino, D. Summa, L. Muccioli, C. Zannoni, D. B. Amabilino, J. Veciana, J. Am. Chem. Soc. 2011, 133, 6603.

[9] D. Klar, A. Candini, L. Joly, S. Klyatskaya, B. Krumme, P. Ohresser, J.-P. Kappler, M. Ruben, H. Wende, Dalton Trans. 2014, 43, 10686.

[10] M. Mannini, F. Bertani, C. Tudisco, L. Malavolti, L. Poggini, K. Misztal, D. Menozzi, A. Motta, E. Otero, P. Ohresser, P. Sainctavit, G. G. Condorelli, E. Dalcanale, R. Sessoli, Nat. Commun. 2014, 5, 4582.

[11] L. Malavolti, V. Lanzilotto, S. Ninova, L. Poggini, I. Cimatti, B. Cortigiani, L. Margheriti, D. Chiappe, E. Otero, P. Sainctavit, F. Totti, A. Cornia, M. Mannini, R. Sessoli, Nano Lett. 2015, 15, 535.

[12] R. Westerström, A.-C. Uldry, R. Stania, J. Dreiser, C. Piamonteze, M. Muntwiler, F. Matsui, S. Rusponi, H. Brune, S. Yang, A. Popov, B. Büchner, B. Delley, T. Greber, Phys. Rev. Lett. 2015, 114, 087201.

[13] J. Dreiser, J. Phys. Condens. Matter 2015, 27, 183203.

[14] N. Ishikawa, M. Sugita, T. Ishikawa, S. Koshihara, Y. Kaizu, J. Am. Chem. Soc. 2003, 125, 8694.

[15] N. Ishikawa, Polyhedron 2007, 26, 2147. 
[16] J. D. Rinehart, M. Fang, W. J. Evans, J. R. Long, Nat. Chem. 2011, $3,538$.

[17] X. Jiang, R. Wang, R. M. Shelby, R. M. Macfarlane, S. R. Bank J. S. Harris, S. S. P. Parkin, Phys. Rev. Lett. 2005, 94, 056601.

[18] I. G. Rau, S. Baumann, S. Rusponi, F. Donati, S. Stepanow, L. Gragnaniello, J. Dreiser, C. Piamonteze, F. Nolting, S. Gangopadhyay, O. R. Albertini, R. M. Macfarlane, C. P. Lutz, B. A. Jones, P. Gambardella, A. J. Heinrich, H. Brune, Science 2014 344, 988.

[19] S. Schintke, S. Messerli, M. Pivetta, F. Patthey, L. Libioulle, M. Stengel, A. De Vita, W.-D. Schneider, Phys. Rev. Lett. 2001, 87 276801.

[20] M. Gonidec, E. S. Davies, J. McMaster, D. B. Amabilino, J. Veciana, J. Am. Chem. Soc. 2010, 132, 1756.

[21] L. Malavolti, M. Mannini, P.-E. Car, G. Campo, F. Pineider, R. Sessoli, J. Mater. Chem. C 2013, 1, 2935.

[22] N. Ishikawa, M. Sugita, W. Wernsdorfer, Angew. Chem. Int. Ed. 2005, 44, 2931.

[23] F. Branzoli, P. Carretta, M. Filibian, M. J. Graf, S. Klyatskaya, M. Ruben, F. Coneri, P. Dhakal, Phys. Rev. B 2010, 82, 134401.

[24] A. Lodi Rizzini, C. Krull, T. Balashov, J. J. Kavich, A. Mugarza, P. S. Miedema, P. K. Thakur, V. Sessi, S. Klyatskaya, M. Ruben, S. Stepanow, P. Gambardella, Phys. Rev. Lett. 2011, 107, 177205.
[25] A. Lodi Rizzini, C. Krull, T. Balashov, A. Mugarza, C. Nistor, F. Yakhou, V. Sessi, S. Klyatskaya, M. Ruben, S. Stepanow, P. Gambardella, Nano Lett. 2012, 12, 5703.

[26] J. Dreiser, C. Wäckerlin, M. E. Ali, C. Piamonteze, F. Donati, A. Singha, K. S. Pedersen, S. Rusponi, J. Bendix, P. M. Oppeneer, T. A. Jung, H. Brune, ACS Nano 2014, 5, 4662.

[27] T. Komeda, H. Isshiki, J. Liu, Y.-F. Zhang, N. Lorente, K. Katoh, B. K. Breedlove, M. Yamashita, Nat. Commun. 2011, 2 217.

[28] S. Joshi, F. Bischoff, R. Koitz, D. Ecija, K. Seufert, A. P. Seitsonen, J. Hutter, K. Diller, J. I. Urgel, H. Sachdev, J. V. Barth, W. Auwärter ACS Nano 2014, 8, 430.

[29] J. Dreiser, R. Westerström, C. Piamonteze, F. Nolting, S. Rusponi, H. Brune, S. Yang, A. Popov, L. Dunsch, T. Greber, Appl. Phys. Lett. 2014, 105, 032411.

[30] A. Zaleski, J. Wrona, M. Czapkiewicz, W. Skowroński, J. Kanak, T. Stobiecki, J. Appl. Phys. 2012, 111, 033903.

[31] S. Yuasa, A. Fukushima, H. Kubota, Y. Suzuki, K. Ando, Appl. Phys. Lett. 2006, 89, 042505.

[32] C. Piamonteze, U. Flechsig, S. Rusponi, J. Dreiser, J. Heidler, M. Schmidt, R. Wetter, M. Calvi, T. Schmidt, H. Pruchova, J. Krempasky, C. Quitmann, H. Brune, F. Nolting, J. Synchrotron Radiat. 2012, 19, 661. 


\section{ADVANCED}

\section{Supporting Information}

for Adv. Mater., DOI: 10.1002/adma.201506305

Giant Hysteresis of Single-Molecule Magnets Adsorbed on a Nonmagnetic Insulator

Christian Wäckerlin, Fabio Donati, Aparajita Singha, Romana Baltic, Stefano Rusponi, Katharina Diller, François Patthey, Marina Pivetta, Yanhua Lan, Svetlana Klyatskaya, Mario Ruben, Harald Brune, and Jan Dreiser* 


\section{Supporting Information for}

\section{“Giant Hysteresis of Single-Molecule Magnets Adsorbed on a Nonmagnetic Insulator"}

by Christian Wäckerlin, Fabio Donati, Aparajita Singha, Romana Baltic, Stefano Rusponi, Katharina Diller, François Patthey, Marina Pivetta, Yanhua Lan, Svetlana Klyatskaya, Mario Ruben, Harald Brune and Jan Dreiser

[*] Dr. Jan Dreiser Swiss Light Source, Paul Scherrer Institut

CH-5232 Villigen PSI (Switzerland)

E-mail: jan.dreiser@psi.ch

Dr. Christian Wäckerlin, Dr. Fabio Donati, Mrs. Aparajita Singha, Ms. Romana Baltic, Dr. Stefano Rusponi, Dr. Katharina Diller, Dr. François Patthey, Dr. Marina Pivetta, Prof. Harald Brune, Dr. Jan Dreiser Institute of Physics (IPHYS), Ecole Polytechnique Fédérale de Lausanne (EPFL)

Station 3, CH-1015 Lausanne (Switzerland)

Dr. Yanhua Lan, Dr. Svetlana Klyatskaya, Prof. Mario Ruben Institute of Nanotechnology, Karlsruhe Institute of Technology (KIT) D-76344 Eggenstein-Leopoldshafen (Germany)

Prof. Mario Ruben

Institut de Physique et Chimie des Matériaux (IPCMS), Université de Strasbourg F-67034 Strasbourg (France)

\section{Contents}

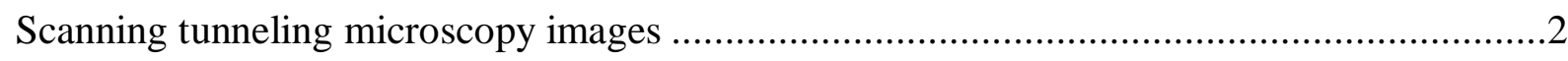

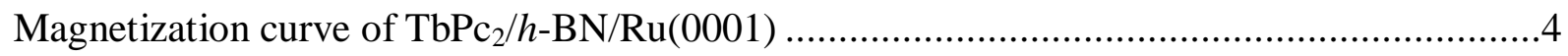

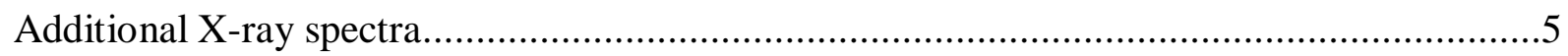

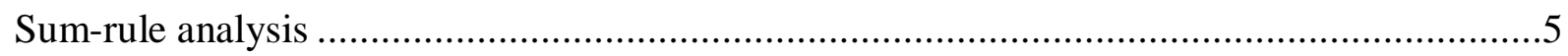

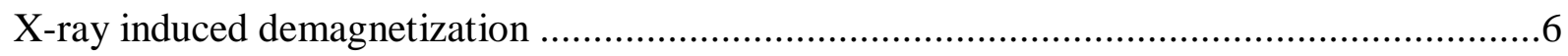

Magnetization curves as a function of the $\mathrm{MgO}$ layer thickness and the $\mathrm{TbPc}_{2}$ coverage.........7

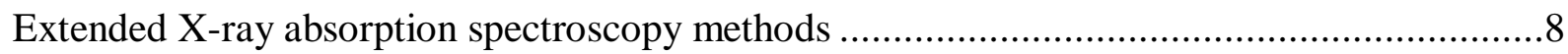

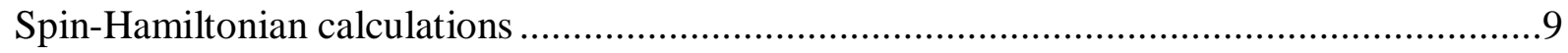

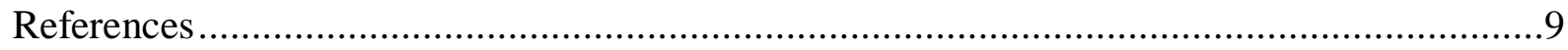




\section{Scanning tunneling microscopy images}

The scanning tunneling microscopy (STM) images were recorded in constant current mode at 4.7 K using a W tip. ${ }^{[1]} \mathrm{STM}$ images of $\mathrm{TbPc}_{2}$ on $\mathrm{Ag}(100)$ and on one monolayer (ML) thick $\mathrm{MgO}$ films were acquired. A large scale STM image of $\mathrm{TbPc}_{2}$ on $\mathrm{MgO} / \mathrm{Ag}(100)$, a model explaining the assembly of $\mathrm{TbPc}_{2}$ on $\mathrm{MgO}$, and an $\mathrm{STM}$ image of $\mathrm{TbPc}_{2} / \mathrm{Ag}(100)$ are shown in Figures S1, S2, and S3, respectively. Molecular fragments, such as TbPc or metal-free Pc molecules, have an abundance of less than $0.1 \%$.

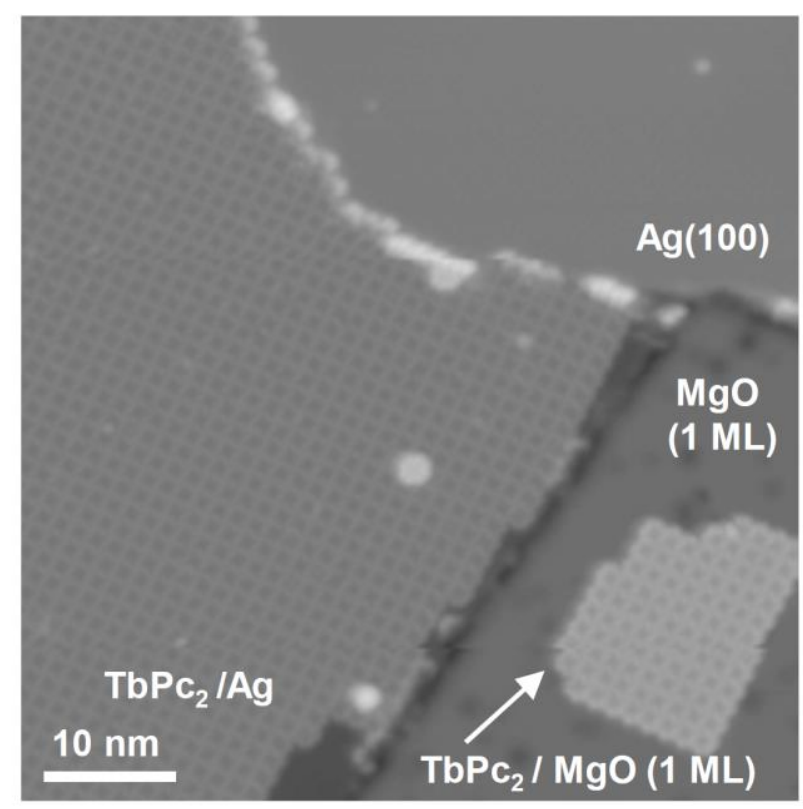

Figure S1 STM overview image showing $\mathrm{Ag}(100), \mathrm{TbPc}_{2} / \mathrm{Ag}(100), 1 \mathrm{ML} \mathrm{MgO} / \mathrm{Ag}(100)$ and $\mathrm{TbPc}_{2} / 1 \mathrm{ML}$ $\mathrm{MgO} / \mathrm{Ag}(100)(+2 \mathrm{~V}, 20 \mathrm{pA})$. The SMMs self-assemble into large islands on $\mathrm{Ag}(100)$ and into smaller islands on $\mathrm{MgO}$. 

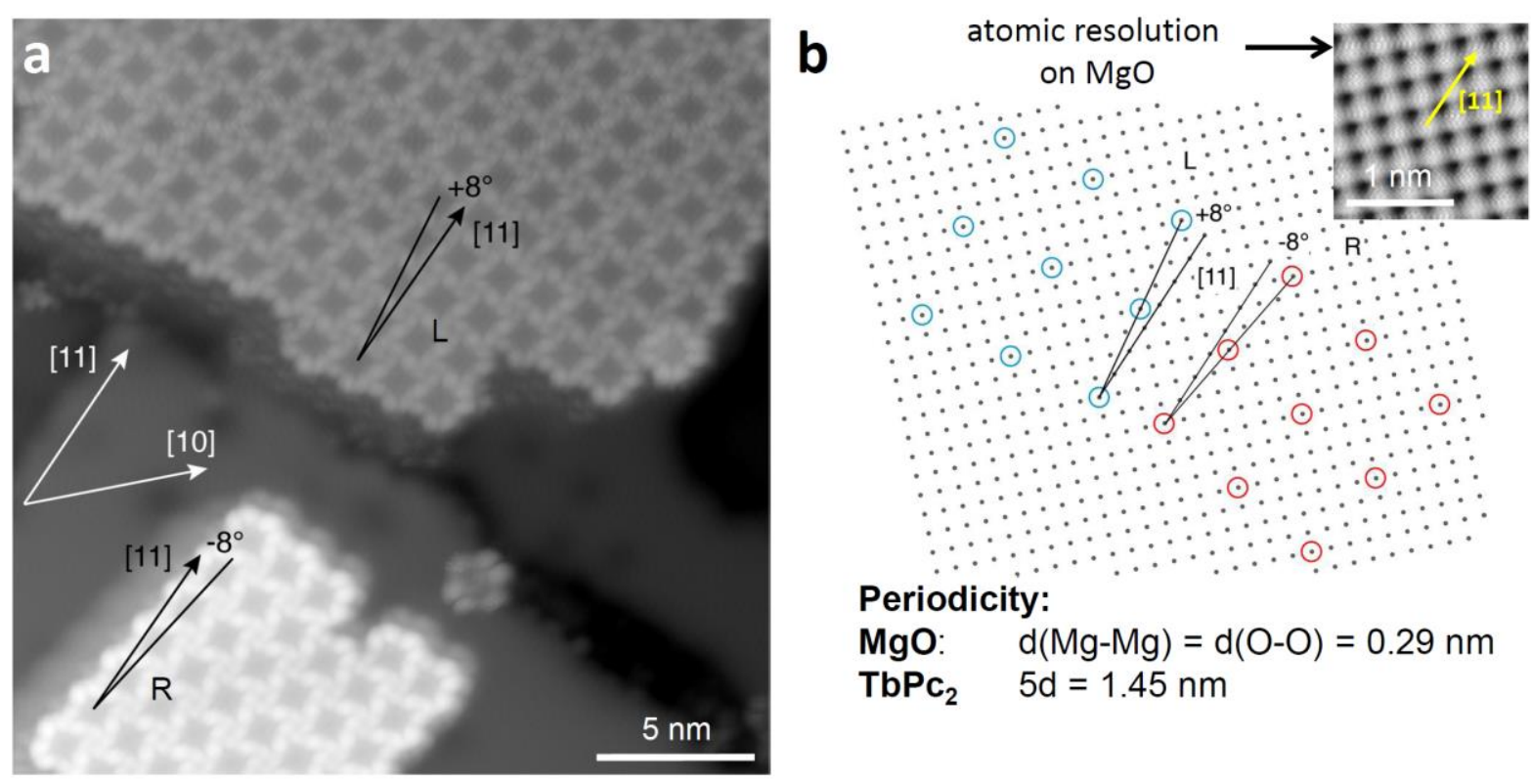

\section{Periodicity:}

MgO: $\quad \mathrm{d}(\mathrm{Mg}-\mathrm{Mg})=\mathrm{d}(\mathrm{O}-\mathrm{O})=0.29 \mathrm{~nm}$

$\mathrm{TbPc}_{2} \quad 5 \mathrm{~d}=1.45 \mathrm{~nm}$

Figure S2 (a) Two mirror domains of the $\mathrm{TbPc}_{2}$ islands which are rotated by $\pm \mathbf{8}^{\circ}$ with respect to the [11] direction of the $\mathrm{MgO}$ lattice and the $\operatorname{Ag}(100)$ substrate $^{[2]}(+2 \mathrm{~V}, 50 \mathrm{pA}$ ). The shadows around the islands result from a slight double tip. Note that commensurability is also possible along the [10] directions. Indeed we observed some islands aligned along [10]. (b) Sketch of the molecular and of the underlying $\mathrm{MgO}$ lattice for both rotational domains. The inset shows atomic resolution on the $\mathrm{MgO}$ layer $(-20 \mathrm{mV}, 2.7 \mathrm{nA})$.

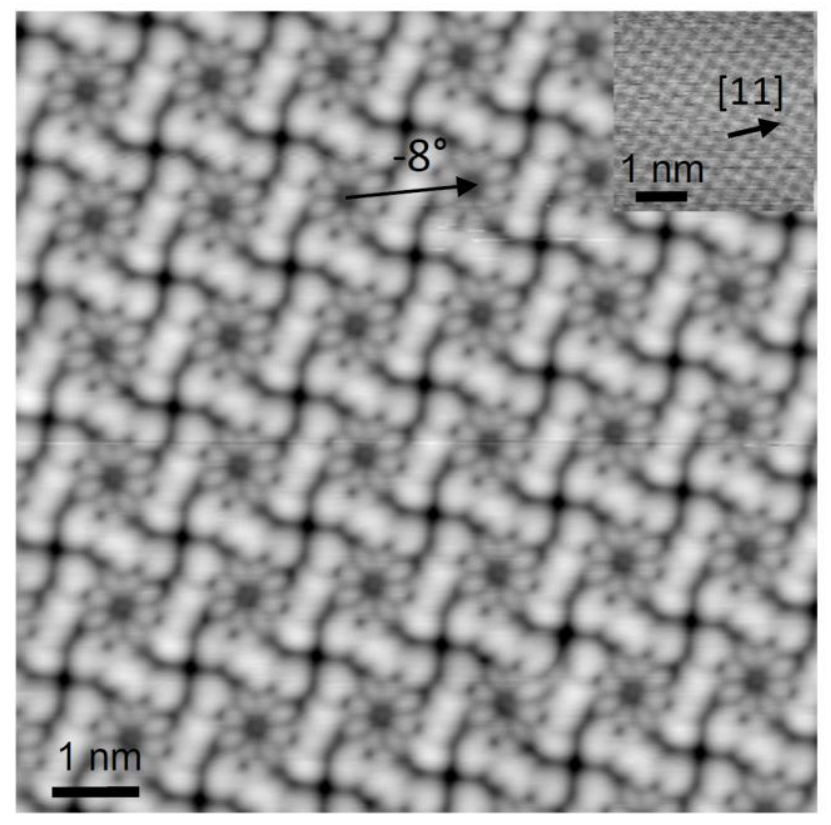

Figure S3 High-resolution STM image of $\mathrm{TbPc}_{2}$ on $\mathrm{Ag}(100)$. Like on $\mathrm{MgO}$, two $\pm \mathbf{8}^{\circ}$ mirror domains of the $\mathrm{TbPc}_{2}$ islands are observed. The STM image shows one $-\mathbf{8}^{\circ}$ domain and resolves the internal structure of the molecules $(-0.8 \mathrm{~V}, 20 \mathrm{pA}) .^{[3-6]}$ The inset shows atomic resolution on the $\mathrm{Ag}(100)$ surface $(-3 \mathrm{mV}, 10 \mathrm{nA})$. 


\section{Magnetization curve of $\mathrm{TbPc}_{2} / h-\mathrm{BN} / \mathrm{Ru}(0001)$}

The hexagonal boron nitride $(h-\mathrm{BN})$ was prepared according to the procedure described in ref. [7], i.e., exposure to borazine $\left(7 \times 10^{-7} \mathrm{mbar}\right)$ while annealing to $1030 \mathrm{~K}$ for 3 minutes and post-annealing for 1 minute. At an X-ray flux $\Phi_{0}$, in normal incidence and at a ramping speed of $2 \mathrm{~T} / \mathrm{min}$ a moderate opening of the hysteresis is observed (Figure S4). The corresponding X-ray spectra are shown in Figure S5c.

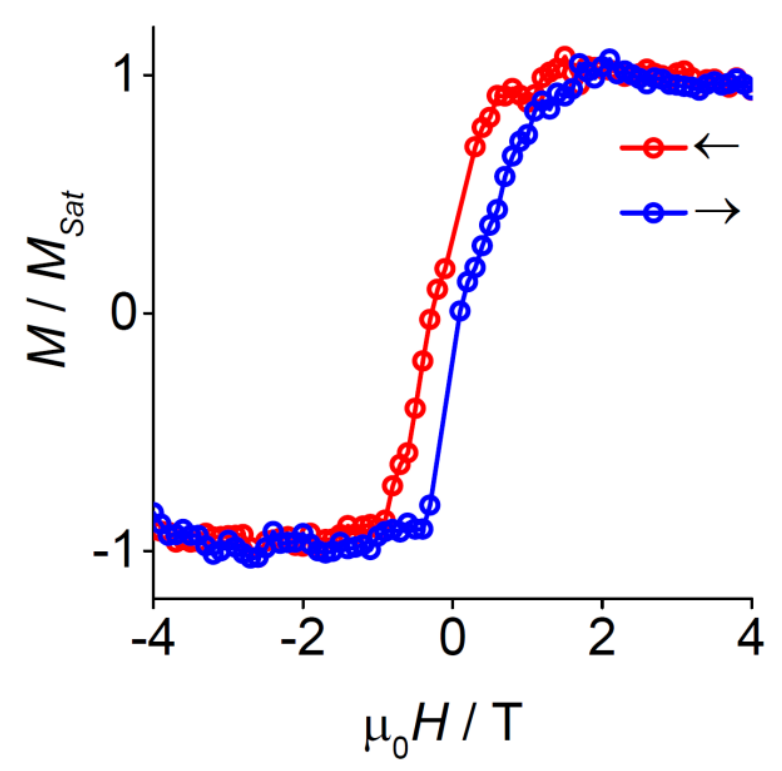

Figure S4 Magnetization curves of $0.3 \mathrm{ML} \mathrm{TbPc}_{2} / h-\mathrm{BN} / \mathrm{Ru}(0001)$ (normal incidence, $3 \mathrm{~K}, \mathrm{X}$-ray flux $\boldsymbol{\Phi}_{\mathbf{0}}, 2$ $\mathrm{T} / \mathrm{min})$. The area of the opening is $56 \mathrm{~J} \mathrm{~mol}^{-1}$ and thus $\sim 4 \times$ larger than that of $\mathrm{TbPc}_{2} / \mathrm{Ag}(100)$ and $\sim 2 \times$ larger than the hysteresis area of the $\mathrm{TbPc}_{2}$ multilayer. Yet, it is $2.5 \times$ smaller than the area of $\mathrm{TbPc}_{2} / \mathrm{MgO} / \mathrm{Ag}(100)$ at the same X-ray flux. 


\section{Additional X-ray spectra}

Figure S5 shows X-ray spectra of multilayer $\mathrm{TbPc}_{2} / \mathrm{MgO} / \mathrm{Ag}(100), \mathrm{TbPc}_{2} / \mathrm{Ag}(100), \mathrm{TbPc}_{2} / h$ $\mathrm{BN} / \mathrm{Ru}(0001)$. The spectra including the ones of $\mathrm{TbPc}_{2} / \mathrm{MgO} / \mathrm{Ag}(100)$ (Figure 2) are very similar and demonstrate that in all cases the molecules adsorb flat on the surfaces with no observable differences in the ligand field acting on the $\mathrm{Tb}$ ion.
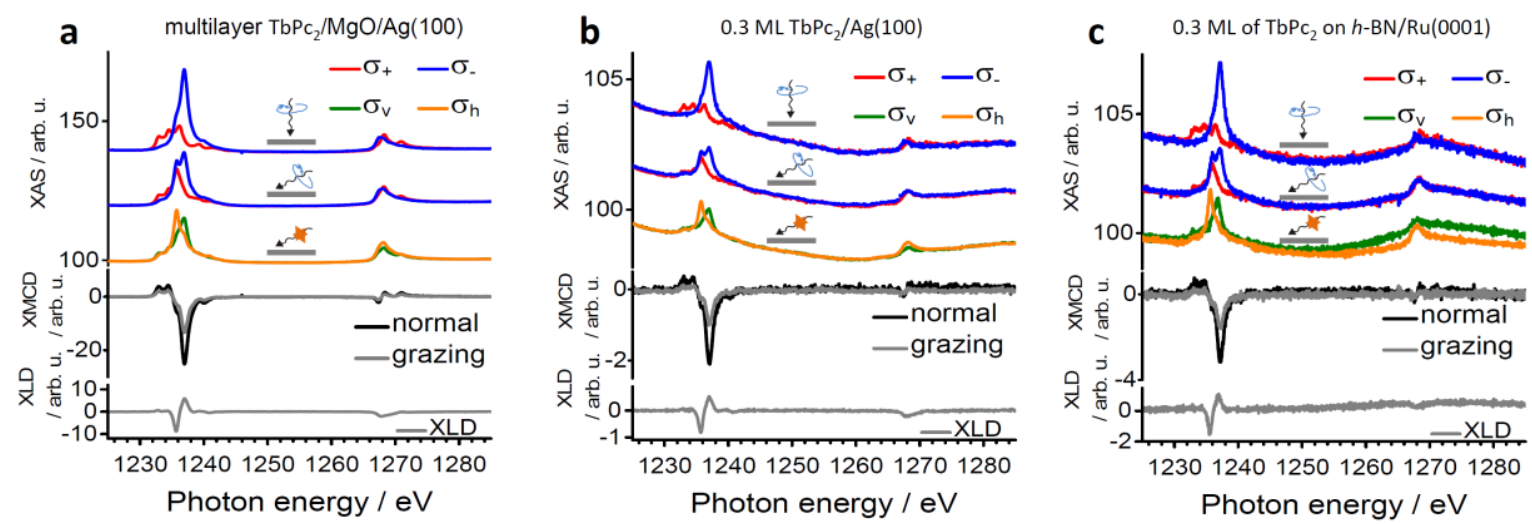

Figure $\mathrm{S5} \mathrm{Tb} \mathrm{M}_{4,5}$ spectra recorded at $3 \mathrm{~K}$ on (a) a multilayer ( 3 ML) of $\mathrm{TbPc}_{2} / \mathrm{MgO} / \mathrm{Ag}(100)$, (b) $0.3 \mathrm{ML}$ $\mathrm{TbPc}_{2} / \mathrm{Ag}(100)$ and (c) $0.3 \mathrm{ML}$ of $\mathrm{TbPc}_{2}$ on $h-\mathrm{BN} / \mathrm{Ru}(0001)$. The XLD was recorded in grazing incidence at 50 $\mathrm{mT}$, and the XMCD was measured in normal $\left(\boldsymbol{\theta}=\mathbf{0}^{\circ}\right)$ and grazing $\left(\boldsymbol{\theta}=\mathbf{6 0}^{\circ}\right)$ incidence at $6.8 \mathrm{~T}$. No background was subtracted. The XLD spectrum is identical to that obtained on the submonolayer of $\mathrm{TbPc}_{2} / \mathrm{MgO}$ (Figure 2) indicating that the molecules adsorb flat in all cases. The same arbitrary units are used within a), b) and c), respectively.

\section{Sum-rule analysis}

The spin and orbital magnetic moments were obtained by application of the XMCD sum rules $^{[8-10]}$ taking into account a number of holes $n_{\mathrm{h}}=6$ for $\mathrm{Tb}(\mathrm{III})$ (Table S1). Values of $\left\langle S_{z}\right\rangle$ $=\left\langle S_{\text {eff }}\right\rangle-3\left\langle T_{z}\right\rangle$ are obtained from the effective spin angular momentum $\left\langle S_{\text {eff }}\right\rangle$ by correction for the magnetic dipole contribution $\left\langle T_{z}\right\rangle$. Here, we use the atomic value $\left\langle T_{z}\right\rangle \mid\left\langle S_{z}\right\rangle=-0.082,{ }^{9}$ while noting that this correction is very close to that obtained from multiplet calculations. ${ }^{[12]}$ 
Table S1 Values of the spin and orbital magnetic moments obtained from sum-rule analyses of the Tb X-ray spectra obtained for $\sim 0.3 \mathrm{ML} \mathrm{TbPc}_{2} / \mathrm{MgO}$ at $6.8 \mathrm{~T}$ and $3 \mathrm{~K}$.

\begin{tabular}{|c|c|c|c|c|}
\hline $\begin{array}{c}\text { Orientation } \\
\boldsymbol{\theta}\end{array}$ & $\begin{array}{c}\mathbf{2}\left\langle S_{\text {eff }}>\right. \\
(\hbar)\end{array}$ & $\begin{array}{c}\mathbf{2}\left\langle S_{z}>\right. \\
(\hbar)\end{array}$ & $\begin{array}{c}\left\langle\boldsymbol{L}_{z}\right\rangle \\
(\hbar)\end{array}$ & $\begin{array}{c}\mathbf{2}\left\langle S_{z}>+\left\langle\boldsymbol{L}_{z}\right\rangle\right. \\
(\hbar)\end{array}$ \\
\hline $0^{\circ}$ & $3.9 \pm 0.2$ & $5.2 \pm 0.3$ & $2.9 \pm 0.2$ & $8.1 \pm 0.3$ \\
\hline $60^{\circ}$ & $2.0 \pm 0.2$ & $2.7 \pm 0.3$ & $1.5 \pm 0.2$ & $4.2 \pm 0.3$ \\
\hline
\end{tabular}

\section{$\mathrm{X}$-ray induced demagnetization}

The cross section $\sigma=0.21 \pm 0.05 \mathrm{~nm}^{2}=(2.1 \pm 0.5) \times 10^{9}$ barn is two orders of magnitude higher than the $\mathrm{X}$-ray absorption cross section at the $\mathrm{Tb} \mathrm{M}_{5}$ edge $\left(\sigma_{\mathrm{M} 5} \cong 2.7 \times\right.$ $10^{7}$ barn). ${ }^{[13]}$ This points to a process that is much more efficient ${ }^{[14]}$ than the direct X-ray excitation of the $\mathrm{Tb}$ ions, suggesting that the molecules are demagnetized via secondary electrons created after the X-ray absorption.

In the following we will falsify explanations of the observed X-ray induced demagnetization by homogeneous heating of the sample by X-ray irradiation. Thermal relaxation with the direct process with $\tau^{-1} \propto T$ would require a temperature increase by $\sim 15 \mathrm{~K} / \Phi_{0}$ which is inconsistent with the low power $\left(\sim 7 \mu \mathrm{W} \mathrm{mm} \mathrm{mm}^{-2} / \Phi_{0}\right)$ of the X-rays. Furthermore, the linear increase of the magnetization relaxation rate with the X-ray flux as seen in Figure $3 b$ is not compatible with a sample heating into a temperature regime in which the Orbach process dominates. Note that the observations are not related to radiation damage, as the same magnetic hysteresis was observed before and after long-time exposure to a high X-ray flux. Since the observed magnetization relaxation time depends on the X-ray flux, we have recorded hysteresis loops on $\mathrm{TbPc}_{2} / \mathrm{MgO}$ for different $\mathrm{X}$-ray fluxes (Figure S6a), revealing significantly narrower openings at high fluxes. Remarkably, even at the highest X-ray flux of $10.8 \Phi_{0}$ the observed hysteresis is still widely open up to $3 \mathrm{~T}$, wider than any hysteresis reported so far on a subML of SMMs. The equilibrium magnetization as determined by spinHamiltonian calculations is shown for comparison.

For $\mathrm{TbPc}_{2}$ on $\mathrm{Ag}(100)$ and for the multilayer (Figure S6b,c) we observe less flux dependence in the magnetization curves. This is expected for a short intrinsic relaxation time rendering the $\mathrm{X}$-ray induced demagnetization in the present range of $\mathrm{X}$-ray fluxes less important. We 
attribute the change in magnetization of $\mathrm{TbPc}_{2} / \mathrm{Ag}(100)$ between $0.3 \mathrm{~T}$ and $1 \mathrm{~T}$ at high $\mathrm{X}$-ray fluxes to the X-ray induced demagnetization.

Since the hysteresis loop is narrow the magnetization curves are suitable to verify the sample temperature by comparing the experimental data with the equilibrium magnetization obtained by the spin-Hamiltonian calculations with $T=3 \mathrm{~K}$. The good overall agreement for all X-ray fluxes indicates, in addition to the arguments given before, that the sample is not significantly warmed up at high X-ray fluxes.
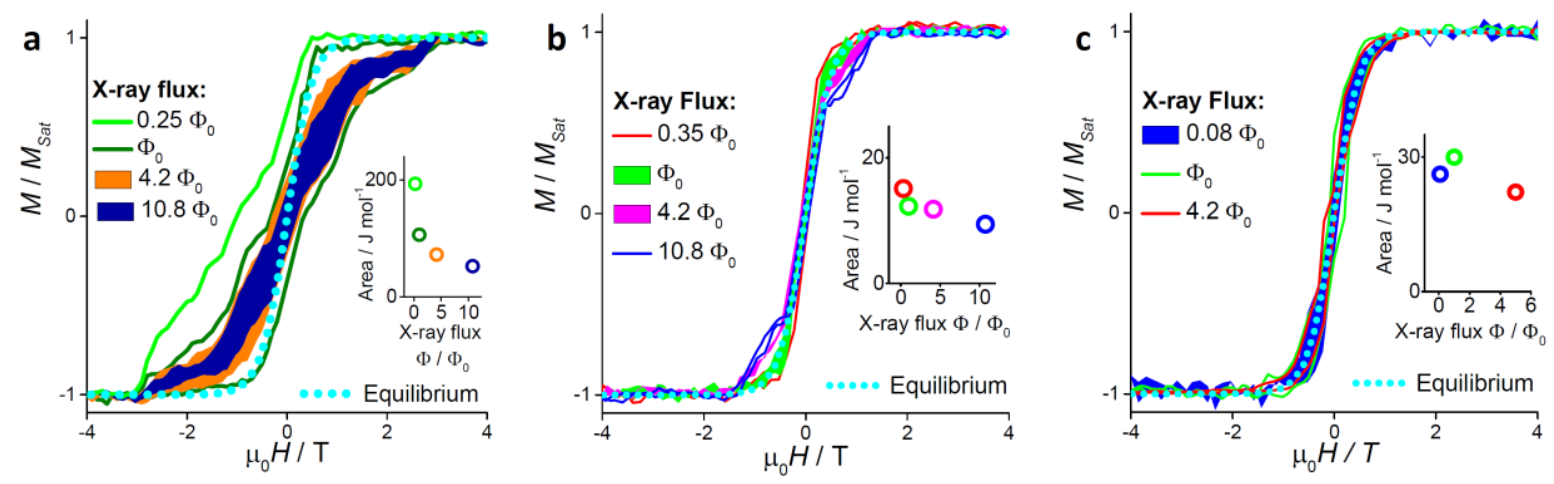

Figure S6 X-ray flux dependent magnetization curves (normal incidence, $3 \mathrm{~K}, 2 \mathrm{~T} / \mathrm{min}$ ) on (a) $\sim 0.3 \mathrm{ML}^{\mathrm{TbPc}} \mathrm{C}_{2}$ on 2.6 ML MgO, the $0.25 \boldsymbol{\Phi}_{\mathbf{0}}$ data is from Figure $1 \mathrm{~d}$, (b) $\sim 0.3 \mathrm{ML} \mathrm{TbPc}_{2} / \mathrm{Ag}(100)$ and on (c) the multilayer $\sim 3$ $\mathrm{ML} \mathrm{TbPc}_{2} / \mathrm{MgO} / \mathrm{Ag}(100)$.

\section{Magnetization curves as a function of the MgO layer thickness and the $\mathrm{TbPc}_{2}$ coverage}

We have obtained $\mathrm{Tb}$ hysteresis loops for varying thicknesses of the $\mathrm{MgO}$ film (Figure S7). On the thinnest layer $(2.6 \mathrm{ML})$, the area of the hysteresis loop is $30 \%$ smaller than on the thickest (4.8 ML), suggesting that the $\mathrm{TbPc}_{2}$ molecules are indeed better protected from scattering with $\mathrm{Ag}$ conduction electrons by thicker $\mathrm{MgO}$ films. It is, however, difficult to draw quantitative conclusions from the results shown in Figure S7 because of the coexistence of several $\mathrm{MgO}$ thicknesses intrinsic to that system. ${ }^{[15,16]}$ 


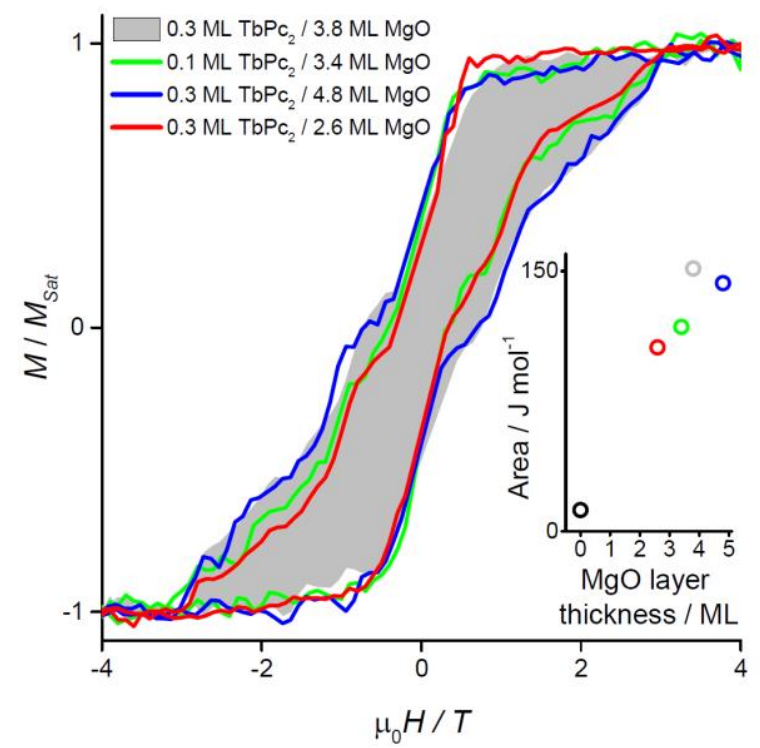

Figure S7 MgO-thickness dependent magnetization curves of $\mathrm{TbPc}_{2}$ on $\mathrm{MgO}$ (normal incidence, $3 \mathrm{~K}, \mathrm{X}$-ray flux $\left.\boldsymbol{\Phi}_{\mathbf{0}}\right)$. The areas shown in the inset quantify the opening of the hysteresis loop. The point at $0 \mathrm{ML} \mathrm{MgO}$ thickness corresponds to the hysteresis of the molecules directly on $\operatorname{Ag}(100)$. Within the scatter of the data we observe no strong influence of the molecular coverage (0.1 ML data point vs. $0.3 \mathrm{ML}$ data points) on the opening. This is consistent with the initial lateral growth of extended 2D domains of self-assembled SMMs.

\section{Extended X-ray absorption spectroscopy methods}

The X-ray absorption measurements were performed in normal $\left(\theta=0^{\circ}\right)$ and grazing $(\theta=$ $60^{\circ}$ ) incidence to characterize the out-of-plane and in-plane magnetization of the SMMs, respectively. All measurements were done in total electron yield (TEY) mode. The magnetic field was applied parallel to the X-ray beam direction. X-rays with linear polarization $\sigma^{\mathrm{v}}$ have their oscillating electric field in the sample plane in both normal and grazing incidence of the beam. A defocused beam $\left(\sim 0.3 \times 1.2 \mathrm{~mm}^{2}\right.$ spot size $)$ was used. The magnetization curves $M(H)$ were obtained by measuring the TEY difference between the $\mathrm{Tb} \mathrm{M}_{5}$ edge and the preedge in an alternating fashion while continuously sweeping the magnetic field. The field sweeps at a rate of $2 \mathrm{~T} / \mathrm{min}$ were performed for the two circular polarizations. In addition, it took $30 \mathrm{~s}$ at zero field for the magnet to switch polarity. Several curves were recorded in order to improve the signal-to-noise ratio. The areas of the magnetization curves were calculated using the value of the $\mathrm{Tb}$ magnetic moment determined by the sum-rule analysis $\left(M_{S a t}=\right.$ $8.15 \mu_{B}$, see below). The magnetization curve in Figure 1d was obtained at an X-ray flux of $0.25 \Phi_{0}$ (with $\Phi_{0}=0.034$ photons $\mathrm{nm}^{-2} \mathrm{~s}^{-1}$ ). It exhibits an area of $193.5 \mathrm{~J} \mathrm{~mol}^{-1}$ which is slightly larger compared to the area of the magnetization curves obtained at a flux of $\Phi_{0}$ on other $\mathrm{TbPc}_{2} / \mathrm{MgO}$ samples. 
The time dependent magnetization was obtained by measuring the XAS signal alternatingly at the edge and the pre-edge for both circular polarizations after sweeping the magnet to the target field at a rate of $2 \mathrm{~T} / \mathrm{min}$. No changes in the X-ray spectra nor in the magnetization curves were observed after illumination even with the highest X-ray flux indicating that there is no observable radiation damage in the molecules.

The ratio of the $\mathrm{M}_{4,5}$ peak areas $v$. the pre-edge background caused by the X-ray absorption of $\mathrm{MgO}$ and the underlying $\operatorname{Ag}(100)$ substrate can be used to estimate the coverage of molecules. For the molecules on $\mathrm{Ag}(100)$ we find a $\mathrm{M}_{4,5}$ peak area vs. pre-edge background ratio of 0.505 for $1 \mathrm{ML}$ (i.e. one full layer of molecules) considering the $\mathrm{M}_{4,5}$ peak area $v s$. pre-edge background ratio of a rare-earth metal reference (Er on $\mathrm{Pt}$ ) and the X-ray attenuation lengths of $\mathrm{Ag}$ and $\mathrm{Pt}$ at the photon energy of the $\mathrm{Tb} \mathrm{M}_{5}$ edge. Considering the very similar observed TEY backgrounds of $\mathrm{Ag}(100)$ and $\mathrm{MgO} / \mathrm{Ag}(100)$, we determine the coverage on $\mathrm{MgO}$ in the same way as on $\mathrm{Ag}(100)$.

\section{Spin-Hamiltonian calculations}

The calculations were performed using a home written MATLAB ${ }^{\circledR}$ code described in ref. [17] in order to obtain the thermodynamic equilibrium magnetization as a function of the applied magnetic field $\boldsymbol{B}$ and the temperature $T$. In order to compare the calculations to the XMCD measurements, the projection of the magnetization onto the X-ray beam direction, identical with the magnetic field direction, is evaluated. The calculations are based on full diagonalization of the spin Hamiltonian and subsequent application of thermal statistics. The Tb magnetic moment is calculated using the spin Hamiltonian $\hat{H}=\sum_{k, q} B_{k}^{q} \hat{O}_{k}^{q}(\mathbf{J})+g_{J} \mu_{\mathrm{B}} \hat{\mathbf{J}} \cdot \mathbf{B}$ with $\hat{O}_{k}^{q}(\mathbf{J})$ the so-called Stevens operators acting on the ground state $J$ manifold and $B_{k}^{q}$ the corresponding coefficients. $\hat{\mathbf{J}}$ is the Tb total (spin and orbital) angular momentum operator. The Stevens operator coefficients, which describe the ligand field acting on the $\mathrm{Tb}(\mathrm{III})$ ion in $\mathrm{TbPc}_{2}$, published in ref. [18], were used taking into account the tabulated Stevens $\alpha, \beta, \gamma$ coefficients.

\section{References}

[1] R. Gaisch, J. K. Gimzewski, B. Reihl, R. R. Schlittler, M. Tschudy, W. D. Schneider, Ultramicroscopy 1992, 42-44, 1621.

[2] S. Schintke, S. Messerli, M. Pivetta, F. Patthey, L. Libioulle, M. Stengel, A. De Vita, W.-D. Schneider, Phys. Rev. Lett. 2001, 87, 276801. 
[3] L. Vitali, S. Fabris, A. M. Conte, S. Brink, M. Ruben, S. Baroni, K. Kern, Nano Lett. 2008, 8, 3364.

[4] T. Komeda, H. Isshiki, J. Liu, Y.-F. Zhang, N. Lorente, K. Katoh, B. K. Breedlove, M. Yamashita, Nat Commun 2011, 2, 217.

[5] J. Schwöbel, Y. Fu, J. Brede, A. Dilullo, G. Hoffmann, S. Klyatskaya, M. Ruben, R. Wiesendanger, Nat. Commun. 2012, 3, 953.

[6] S. Fahrendorf, N. Atodiresei, C. Besson, V. Caciuc, F. Matthes, S. Blügel, P. Kögerler, D. E. Bürgler, C. M. Schneider, Nat. Commun. 2013, 4, DOI 10.1038/ncomms3425.

[7] F. D. Natterer, F. Patthey, H. Brune, Phys. Rev. Lett. 2012, 109, 066101.

[8] B. Thole, P. Carra, F. Sette, G. van der Laan, Phys. Rev. Lett. 1992, 68, 1943.

[9] P. Carra, B. Thole, M. Altarelli, X. Wang, Phys. Rev. Lett. 1993, 70, 694.

[10] Y. Teramura, A. Tanaka, B. T. Thole, T. Jo, J. Phys. Soc. Jpn. 1996, 65, 3056.

[11] M. Gonidec, R. Biagi, V. Corradini, F. Moro, V. De Renzi, U. del Pennino, D. Summa, L. Muccioli, C. Zannoni, D. B. Amabilino, J. Veciana, J. Am. Chem. Soc. 2011, 133, 6603.

[12] S. Stepanow, J. Honolka, P. Gambardella, L. Vitali, N. Abdurakhmanova, T.-C. Tseng, S. Rauschenbach, S. L. Tait, V. Sessi, S. Klyatskaya, M. Ruben, K. Kern, J. Am. Chem. Soc. 2010, 132, 11900.

[13] B. Thole, G. van der Laan, J. Fuggle, G. Sawatzky, R. Karnatak, J.-M. Esteva, Phys. Rev. B 1985, 32, 5107.

[14] J. Dreiser, R. Westerström, C. Piamonteze, F. Nolting, S. Rusponi, H. Brune, S. Yang, A. Popov, L. Dunsch, T. Greber, Appl. Phys. Lett. 2014, 105, 032411.

[15] J. Wollschläger, D. Erdös, H. Goldbach, R. Höpken, K. . Schröder, Thin Solid Films 2001, 400, 1 .

[16] J. Pal, M. Smerieri, E. Celasco, L. Savio, L. Vattuone, M. Rocca, Phys. Rev. Lett. 2014, $112,126102$.

[17] J. Dreiser, C. Wäckerlin, M. E. Ali, C. Piamonteze, F. Donati, A. Singha, K. S. Pedersen, S. Rusponi, J. Bendix, P. M. Oppeneer, T. A. Jung, H. Brune, ACS Nano 2014, 5, 4662.

[18] N. Ishikawa, T. Iino, Y. Kaizu, J. Phys. Chem. A 2002, 106, 9543. 